\title{
Osteogenesis imperfecta (lethal) bones contain types III and V collagens
}

\author{
FM POPE, AC NiCHOLls, CHRISTINE EGGLETON, P NARCISSi, EN HEY, \\ AND JM PARKIN
}

From the Division of Clinical Sciences, Clinical Research Centre, Watford Road, Harrow HAI 3 UJ

and the Children's Department, The Royal Victoria Infirmary, Newcastle-upon-Tyne NEI 4LP, UK

SUMMARY Lethal osteogenesis imperfecta (OI-L) and normal fetal bones contain types I and V collagen with relatively more type $\mathrm{V}$ in OI-L bones. The latter, unlike normal fetal bone, also contain some type III collagen. Such altered collagen ratios could directly produce the bony fragility and radiotranslucency of OI-L bones. Since this is an inherited osteoporosis similar alterations in acquired osteoporoses are also possible.

Osteogenesis imperfecta (OI) is an inherited defect of connective tissue in which bony fragility is associated with generalised connective tissue abnormalities such as hernias, hyperextensible skin, blue sclerae, deafness, and dentinogenesis imperfecta. There is autosomal dominant and recessive inheritance, ${ }^{1}$ and each form is clinically and probably biochemically heterogeneous. ${ }^{2}$ Sillence and Rimoin ${ }^{3}$ have proposed five types of OI, and there are probably more. Abnormalities of collagen chemistry probably cause most or all of them, and diminished type I collagen synthesis by cultured fibroblasts, ${ }^{4}$ inadequate lysine hydroxylation, ${ }^{5}$ altered tissue ratios of type III to type I collagen in skin biopsies, ${ }^{6}$ and failure to produce $\alpha 2$ chains $^{7}$ have been described. The rare lethal or broad-boned variety of OI is particularly severe, causing death in utero or in early infancy. Cranial enlargement, chest wall deformities, short-limbed dwarfism, and curiously undercalcified, broadened, misshapen bones are characteristic of the syndrome. Penttinen et al. ${ }^{4}$ have identified an alteration of collagen ratios produced by cultured skin fibroblasts from such individuals and showed a markedly diminished type I and relatively increased type III collagen production. Muller et al. ${ }^{8}$ described similar patterns in a different sort of patient who certainly had brittle bones but not short-limbed dwarfism.

We have recently detected hitherto undescribed bone collagens from two affected patients with lethal OI.

Received for publication 10 January 1980

\section{Methods}

Postmortem femoral bone samples were obtained from two full-term babies with lethal OI (OI-L). They fulfilled the clinical and radiological criteria of fatal short-limbed dwarfism with abnormally broad, undercalcified bones (Fig. 1). Control bones were obtained from five normal fetuses of between 16 and 39 weeks' gestation. The bones were finely minced with a scalpel and decalcified in $0.5 \mathrm{M}$ EDTA, $\mathrm{pH} 7 \cdot 6$, for 48 hours at $4^{\circ} \mathrm{C}$. After spinning at $2000 \mathrm{~g}$ for 30 minutes and resuspension in $0.5 \mathrm{M}$ acetic acid, pepsin (Worthington) was added to $1 \%$ wet weight of bone and digested for 16 hours at $15^{\circ} \mathrm{C} .9^{9}$ The reaction was stopped by raising the $\mathrm{pH}$ to 8.0 for 30 minutes and then desalted by dialysis against $0.5 \mathrm{M}$ acetic acid and lyophilised. Aliquots were taken for analysis of whole collagen chains and cyanogen bromide peptide mapping. Whole chains were examined on $5 \%$ polyacrylamide gels in tris borate SDS at $\mathrm{pH} 8 \cdot 6,10$ and cyanogen bromide peptides were separated by tube or slab gel electrophoresis using either tris borate SDS at $\mathrm{pH} 8 \cdot 6^{10}$ or SDS phosphate at $\mathrm{pH} \mathbf{7 \cdot 2 . 1 1}$

Bacterial collagenase digestion of whole collagen chains (Clostridial Collagenase BDH) was carried out at $37^{\circ} \mathrm{C}$ for 16 hours in $0.2 \mathrm{M} \mathrm{NH}_{4} \mathrm{HCO}_{3}$ and $\mathrm{CaCl}_{2}$, $\mathrm{pH} 8 \cdot 6 .{ }^{12}$

Type V collagen was prepared from placental membranes using salt precipitation of pepsinised material. ${ }^{13}$ A $4 \%$ salt precipitation of pepsinised membranes separated a supernatant greatly en- 


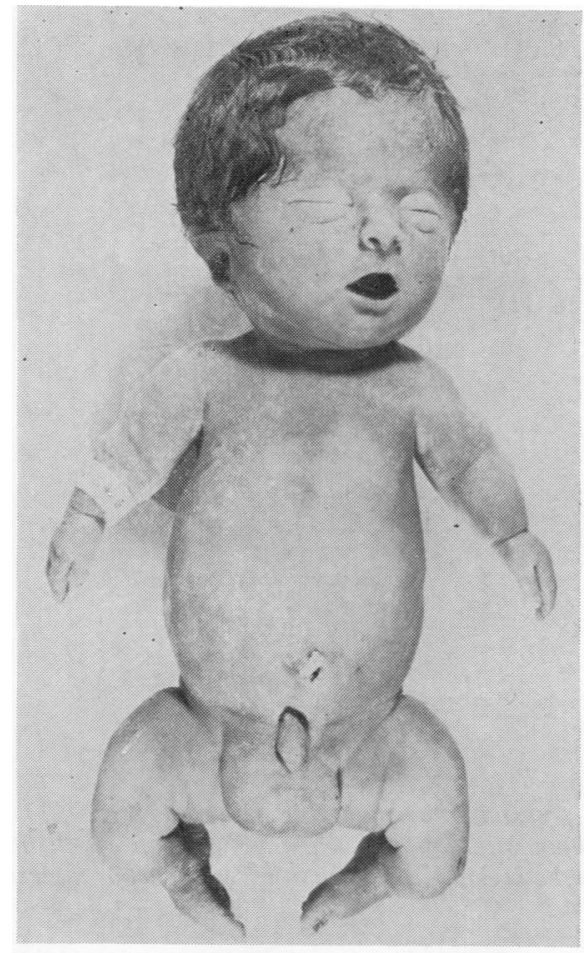

(a)

Fig. 1 Typical clinical and X-ray changes of OI-L.

riched in type $\mathrm{V}$ collagen and a precipitate containing types I and III collagens.

AMINO-ACID ANALYSIS

Samples were dissolved in $6 \mathrm{~N} \mathrm{HCl}$, flushed with $\mathrm{N}_{2}$, and heated in sealed glass tubes at $110^{\circ} \mathrm{C}$ for 24 hours and analysed on a Technicon amino-acid analyser.

\section{Results}

\section{WHOLE COLLAGEN CHAINS}

Pepsin digested OI-L bone consistently showed slower migrating bands than $\alpha 1(\mathrm{I})$ and a mercaptoethanol reducible trimer $(\gamma)$ separable from $\alpha 1(I)$ by interrupted electrophoresis. The latter is specific for type III collagen. ${ }^{14}$ The slower migrating bands from the OI-L bone had two components, an upper $(\alpha B)$ and lower $(\alpha A)$ band, although the $\alpha B$ band predominated. The electrophoretic conditions were arranged such that the reducible $\gamma$ component migrated as an $\alpha$ chain just below the $\alpha \mathrm{B}$ chain and above the $\alpha$ A position. The $\alpha \mathrm{B}$ band from OI-L bone

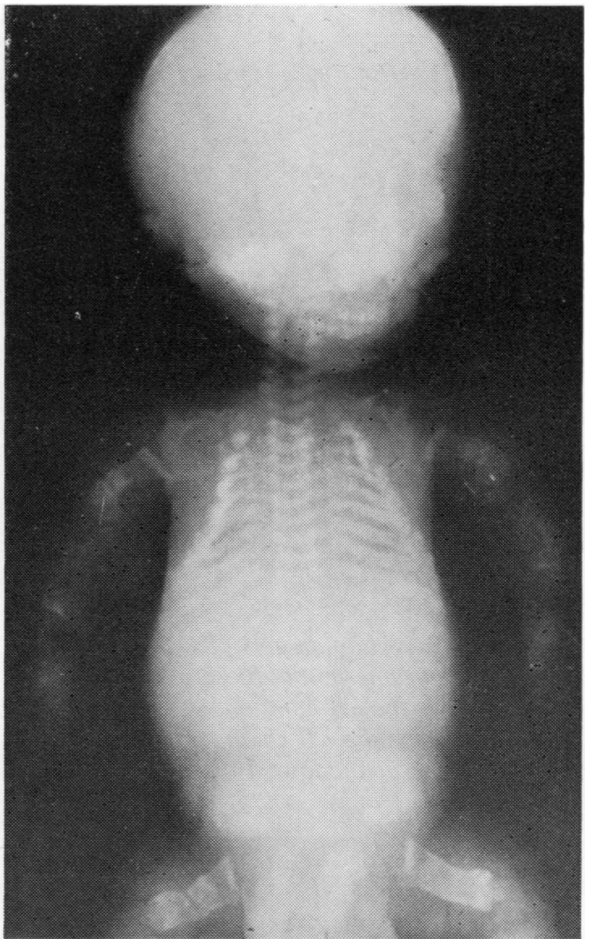

(b)

was present in sufficient amounts to persist at even one-tenth of the normal concentration applied to the gels (Fig. 2). In contrast, the control bones contained lesser amounts, and loading experiments showed rough equivalence between $100 \mu \mathrm{g}$ of pepsinised control and 10-20 $\mu \mathrm{g}$ of OI bone (Fig. 3). Comparison with authentic type $\mathrm{V}$ collagen prepared from human fetal membranes showed that the bands from bone have similar electrophoretic properties to type $\mathrm{V}$ collagen $\alpha \mathrm{A}$ and $\alpha \mathrm{B}$ chains. To confirm that type $\mathrm{V}$ collagen occurs in normal bone, the pepsinised collagens were enriched by precipitation with $4 \%$ $\mathrm{NaCl}$ and showed authentic type $\mathrm{V}$ collagen in the supernatant fraction (Fig. 4). Similar experiments with OI-L bone were restricted by the lack of material.

Since coincident electrophoresis of putative collagenous bands is not proof-positive that they contain collagen, the pepsinised material was treated with bacterial collagenase before electrophoresis. This experiment showed the heavy component to be collagenase sensitive (Fig. 5).

The proportion of collagen solubilised was esti- 


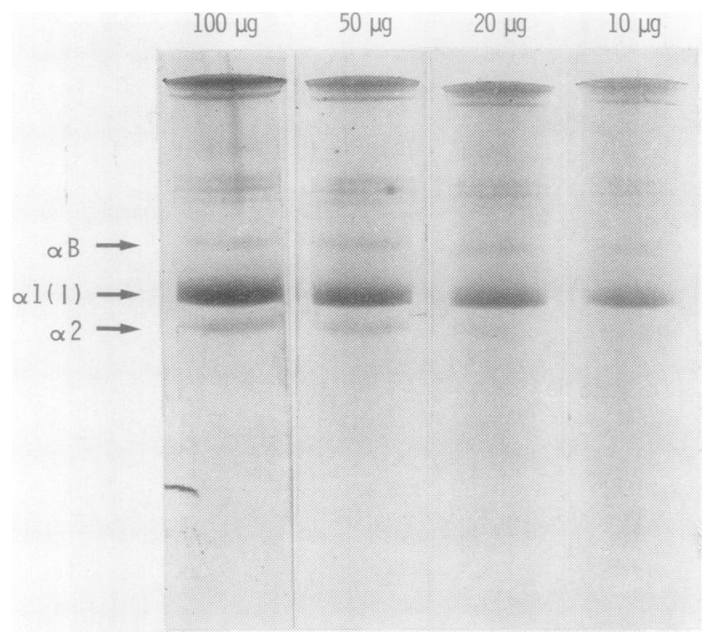

Fig. $25 \%$ polyacrylamide gels of OI-L bone at normal $(100 \mu \mathrm{g})$ to one-tenth $(10 \mu \mathrm{g})$ concentration.

This clearly shows the aB component, and the aA chain is not seen on this gel. The aB chains are sufficiently plentiful to be seen at the lowest concentration gel.

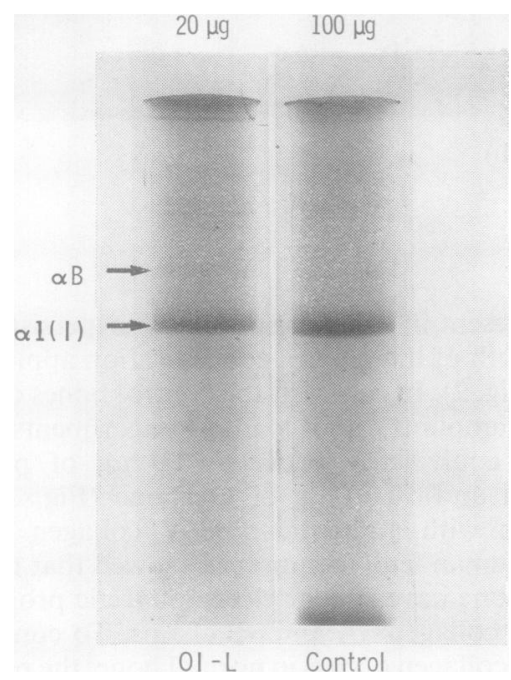

Fig. $35 \%$ polyacrylamide gels showing equivalence between different concentrations of $O I-L$ and control bone. This is to contrast the relative amounts of $a B$ chain which is as plentiful in OI-L bones at one-fifth the loading of the control bone.

mated from the hydroxyproline content of the pepsin soluble and insoluble fractions. In all cases this was greater than $60 \%$.

CYANOGEN BROMIDE CLEAVAGE

Cyanogen bromide cleaved peptides range from
Pope, Nicholls, Eggleton, Narcissi, Hey, and Parkin

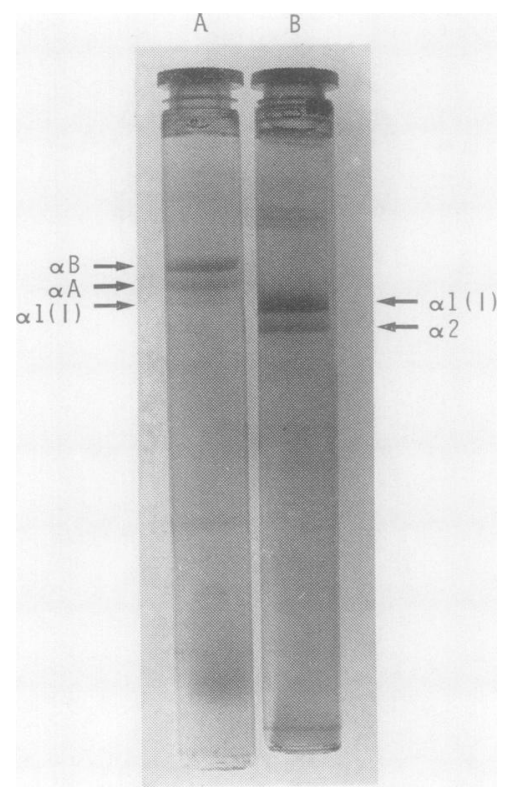

Fig. 4 Pepsinised normal bone showing (A) the supernatant with almost pure type $V a A$ and $a B$ chains and (B) the precipitation with other collagenous material.

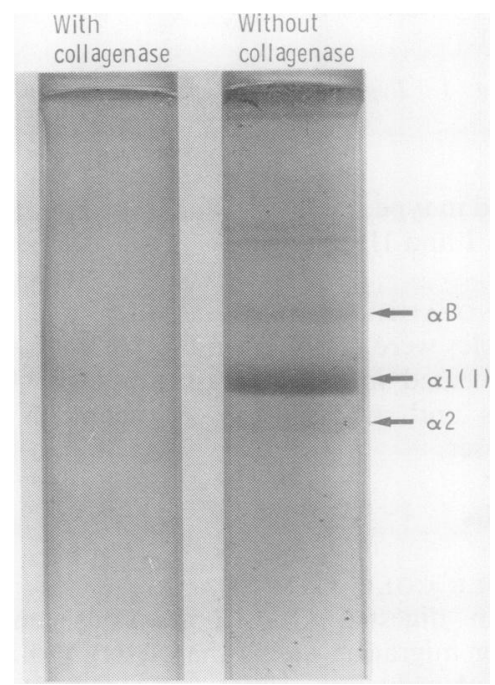

Fig. $55 \%$ polyacrylamide gels of OI-L bone showing that the various bands are collagenase sensitive.

3000 to 22000 daltons molecular weight and are separable by either gel electrophoresis ${ }^{11}$ or column chromatography. ${ }^{15}$ There were distinct differences between the peptide patterns of equivalent amounts of cleaved bone from OI-L and control patients 
separated by the tris borate system at $\mathrm{pH} 8 \cdot 6.10$ Normal bone contained a peptide absent from OI bone (arrow 1, Fig. 6), and OI bone contained a large excess of a low molecular weight peptide, possibly characteristic of type $\mathrm{V}$ collagen (arrow 2, Fig. 6). Further studies are in progress specifically to identify the significance of these peptide differences. Similar changes with a slightly different pattern were visible in the SDS phosphate system. ${ }^{11}$

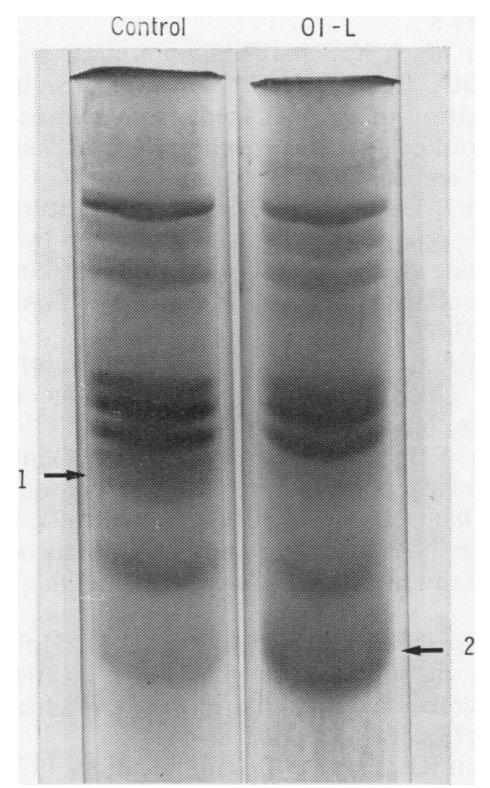

Fig. $6 \quad 10 \%$ polyacrylamide gels of cyanogen bromide peptides showing differences between control and $O I$ bones when run at identical loading concentrations. The differences between the peptide patterns are shown by the arrows.

\section{Discussion}

Collagen is a complex triple helical structural protein which is genetically heterogeneous, and in recent years two, ${ }^{16}$ three, ${ }^{17}$ four, ${ }^{18}$ and then five ${ }^{19}$ types have been recognised. Type $\mathrm{V}$ was identified only in 1976 in fetal membranes by Burgeson et al. ${ }^{19}$ and, although subsequently identified at other sites such as skin, cartilage, lung, and intestine, ${ }^{20}$ has never been described in bone. The chain composition and chromatographic properties of the various collagen types have been summarised in the Table.

Until recently, largely because of Miller's work, bone was thought to contain only type I collagen. ${ }^{21}$ Muller et al. ${ }^{22}$ then observed small amounts of type III collagen occurring in holes within compact bone from a patient with OI tarda. We have definite proof that the poorly calcified and abnormally fragile OI-L bones contain sufficient quantities of a collagen very similar to, or identical with, type $\mathrm{V}$ to be detectable in unconcentrated pepsinised whole material as well as traces of type III collagen. It was barely detected in control fetal bones although it can be concentrated by $4 \%$ salt precipitation, and type III is absent altogether in normal bones. The collagen chains identified as type $\mathrm{V}$ collagen comigrate with authentic type $\mathrm{V}$, are heavier than $\alpha 1$ (I) chains, are collagenase sensitive, and can be prepared from normal fetal bones only by using standard purification methods for type $\mathrm{V}$ fetal membrane collagen. Since it is barely detectable in normal fetuses and is richly present in full-term, OI-L bone, it is tempting to attribute to it a role in the mechanism of fragility. One possibility is that type $\mathrm{V}$ and type III collagens are normally necessary only to the developing fetal bones. Perhaps an increased ratio of type V and III collagens relative to type I interferes with proper bony calcification. Alternatively, type $\mathrm{V}$ collagen is the effect but not the cause of poor calcification and is an inadequate attempt to compensate for diminished type I collagen production. The definite identification of type III collagen in OI-L bone accords with the apparent increase of type III collagen synthesis by cultured skin fibroblasts in this disease, as described by Penttinen et al. ${ }^{4}$ Since type V collagen overlaps with $\alpha 1$ (III) on CMC chromatography, further studies are essential to know whether OI-L fibroblasts also synthesise increased quantities of type $\mathrm{V}$ collagen in addition to type III as Penttinen's published chromatograms do not exclude this possibility; this work is currently in progress.

Further studies are in progress to measure type $\mathrm{V}$ to type I collagen ratios in normal babies throughout

Table Chain composition and properties of genetically distinct collagens

\begin{tabular}{|c|c|c|}
\hline Collagen type & Chain composition & Properties \\
\hline $\begin{array}{l}\text { I } \\
\text { II } \\
\text { III }\end{array}$ & $\begin{array}{l}a 1(\text { I) })_{2} \\
a 1(\text { III })_{3} \\
a 1(\text { III })_{3}\end{array}$ & $\begin{array}{l}a 1, a 2 \text { separate on polyacrylamide gels and carboxymethyl cellulose } \\
a !(I !) \text { runs with } \alpha 1(\mathrm{I}) \text { on gels and CM cellulose } \\
\text { al(III) runs as trimer on gels until reduced with mercaptoethanol, when it runs } \\
\text { with } a 1(\mathrm{I}) ; a 1 \text { (III) runs between } a 1 \text { (I) and } a 2 \text { on } \mathrm{CM} \text { cellulose }\end{array}$ \\
\hline $\begin{array}{l}\text { IV } \\
\text { V }\end{array}$ & $\begin{array}{l}a 1(\mathrm{IV})_{3} \\
a \mathrm{AaB}\end{array}$ & $a A a B$ run behind $a 1(I)$ on gels, between $a 1$ and $\alpha 2$ on $C M$ cellulose \\
\hline
\end{tabular}


pregnancy and early infancy. If type $\mathrm{V}$ normally disappears in late fetal life or early childhood, then its persistence in full-term OI-L bones implies either a causative role or that OI-L bones retain their early fetal state. In any case, the amount of type $\mathrm{V}$ collagen in OI-L bones is prominent but it is detected only with difficulty in normal fetal bones. OI-L is an example of a genetic defect in which brittle osteoporotic bones contain unusual collagen ratios. It is possible that the absence of collagens other than type $I$ in these bones allows such osteoporoses to occur. Similar alterations may also explain other osteoporoses occurring later in life. It is by no means impossible that hormones such as steroids and oestrogen may induce unusual bone collagens and so allow an osteoporotic process to occur. This hypothesis will be easily tested.

\section{References}

${ }^{1}$ McKusick VA. Heritable Disorders of Connective Tissue, 4th ed. St. Louis: Mosby, 1972.

${ }^{2}$ Pope FM. Collagen and osteogenesis imperfecta. Letter, Lancet 1976;1:1024.

3 Sillence DO, Rimoin DL. Classification of osteogenesis imperfecta. Letter, Lancet 1978;1:1041-2.

4 Penttinen RP, Lichtenstein JR, Martin GR, McKusick VA. Abnormal collagen metabolism in cultured cells in osteogenesis imperfecta. Proc Nat Acad Sci USA 1975;72:586-9.

5 Trelstad RL, Rubin D, Gross J. Osteogenesis imperfecta congenita: evidence for a generalised molecular disorder of collagen. Lab Invest 1977;36:501-8.

${ }^{6}$ Sykes BC, Francis MJO, Smith R. Altered relation of two collagen types in osteogenesis imperfecta. $N$ Eng J Med 1977;296:1200-3.

7 Nicholls AC, Pope FM, Schloon H. Biochemical heterogeneity of osteogenesis imperfecta: new variant. Letter, Lancet 1979;1:1193.

${ }^{8}$ Muller PK, Lemmen C, Gay S, Meigel WN. Disturbance in the regulation of the type of collagen synthesised in a form of osteogenesis imperfecta. Europ J Biochem 1975;59:97-104.

${ }^{9}$ Chung E, Miller EJ. Collagen polymorphism: charac- terization of molecules with the chain composition [a1(III)] $]_{3}$ in human tissues. Science 1974;183:1200-1.

${ }^{10}$ Sykes BC, Bailey AJ. Molecular weight heterogeneity of the a-chain sub-units of collagen. Biochem Biophys Res Commun 1971;43:340-5.

${ }^{11}$ Furthmayr H, Timpl R. Characterisation of collagen peptides by sodium dodecylsulfate-polyacrylamide electrophoresis. Analyt Biochem 1971;41:510-6.

${ }^{12}$ Fietzek PP, Rexrodt FW, Wendt P, Stark M, Kuhn K. The covalent structure of collagen: amino-acid sequence of peptide a1-CB6-C2. Eur J Biochem 1972; $30: 163-8$.

13 Von der Mark K. Personal communication, 1978.

${ }^{14}$ Sykes BC, Puddle B, Francis MJO, Smith R. The estimation of two collagens from human dermis by interrupted gel electrophoresis. Biochem Biophys Res Commun 1976;72:1472-80.

${ }^{15}$ Miller EJ, Epstein EH, Jr, Piez KA. Identification of three genetically distinct collagens by cyanogen bromide cleavage of insoluble human skin and cartilage collagen. Biochem Biophys Res Commun 1971; 42:1024-9.

${ }^{16}$ Miller EJ. Isolation and characterization of a collagen from chick cartilage containing three identical $a$ chains. Biochemistry 1971;10:1652-8.

${ }^{17}$ Epstein EH, Jr. [a1(III) $]_{3}$ human skin collagen: release by pepsin digestion and preponderance in fetal life. J Biol Chem 1974;249:3225-31.

${ }^{18}$ Kefalides NA. Structure and biosynthesis of basement membranes. Int Rev Connect Tissue Res 1973;6:63104.

${ }^{19}$ Burgeson RE, El Adli FA, Kaitila II, Hollister DW. Fetal membrane collagens: identification of two new collagen alpha chains. Proc Nat Acad Sci USA 1976;73:2579-83.

${ }^{20}$ Burgeson RE. Personal communication, 1978.

${ }^{21}$ Miller EJ. Biochemical studies on the structure of chick bone collagen. Fed Proc 1969;28:1839-45.

${ }^{22}$ Muller PK, Raisch K, Matzen K, Gay S. Presence of type III collagen in bone from a patient with osteogenesis imperfecta. Eur J Pediatr 1977;125:29-37.

Requests for reprints to: Dr FM Pope, Division of Clinical Sciences, Clinical Research Centre, Watford Road, Harrow, Middlesex HA1 3UJ, UK. 